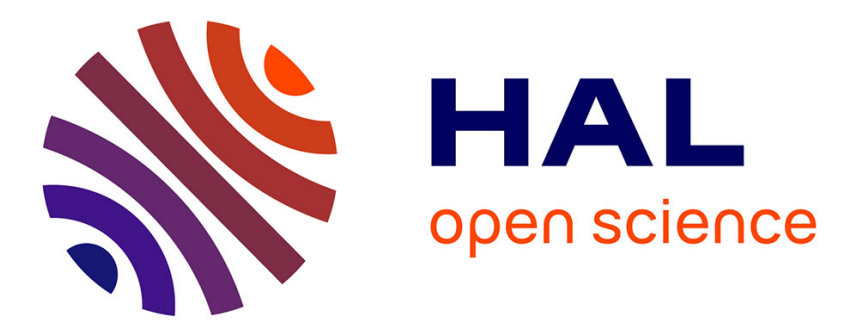

\title{
Shock Modon: A New Type of Coherent Structure in Rotating Shallow Water
}

Noé Lahaye, Vladimir Zeitlin

\section{To cite this version:}

Noé Lahaye, Vladimir Zeitlin. Shock Modon: A New Type of Coherent Structure in Rotating Shallow Water. Physical Review Letters, 2012, 108 (4), pp.044502. 10.1103/physrevlett.108.044502 . hal01115483

\section{HAL Id: hal-01115483 \\ https://hal.science/hal-01115483}

Submitted on 13 Jan 2022

HAL is a multi-disciplinary open access archive for the deposit and dissemination of scientific research documents, whether they are published or not. The documents may come from teaching and research institutions in France or abroad, or from public or private research centers.
L'archive ouverte pluridisciplinaire HAL, est destinée au dépôt et à la diffusion de documents scientifiques de niveau recherche, publiés ou non, émanant des établissements d'enseignement et de recherche français ou étrangers, des laboratoires publics ou privés. 


\title{
Shock Modon: A New Type of Coherent Structure in Rotating Shallow Water
}

\author{
Noé Lahaye ${ }^{1}$ and Vladimir Zeitlin ${ }^{1,2}$ \\ ${ }^{1}$ Laboratoire de Météorologie Dynamique/IPSL, UPMC-ENS, Paris, France \\ ${ }^{2}$ Institut Universitaire de France, \\ 24 rue Lhomond, 75005 Paris, France
}

(Received 11 October 2011; published 26 January 2012)

\begin{abstract}
We show that a new type of coherent structure, a shock modon, exists in a rotating shallow water model at large Rossby numbers. It is a combination of an asymmetric vortex dipole with a stationary hydraulic jump. The structure is long living, despite the energy dissipation by the hydraulic jump, and moving along a circular path. Collisions of shock modons can be elastic, or lead to formation of shock tripoles.
\end{abstract}

PACS numbers: 47.27.De, 47.32.C-, 47.40.Hg, 95.30.Qd

Rotating shallow water (RSW) is a standard conceptual model in geophysical fluid dynamics [1]. It is also relevant for various astrophysical applications, such as dynamics of the solar tachocline [2] and dynamics of the accretion disks [3]. It should be also remembered that the well-known quasigeostrophic (QG) model for large-scale oceanic and atmospheric motions [1], which follows from the RSW equations in the limit of small Rossby numbers $\left(\mathrm{Ro}=\frac{U}{f L}\right.$, where $U$ and $L$ are, respectively, typical velocity and length scales and $f$ is the Coriolis parameter), is equivalent to the Hasegawa-Mima equation [4] for drift waves in plasma.

The famous modon (dipolar steady-moving localized vortex) solutions of the QG equation are long-known [5]. (Note that the so-called beta-plane version of the QG equation - where the meridional gradient of the Coriolis force is taken into account-was used in this paper, although the so-called $f$-plane constant Coriolis parameter version of the modon is also known [6].) The quasigeostrophic equation being an asymptotic limit of RSW equations, a natural question arises about the existence of modons in the full model at finite Rossby numbers. Recently, such an ageostrophic modon solution of the RSW equations was obtained theoretically [7], and found numerically [8] by relaxation of the QG modon which was taken as the initial configuration in the full RSW model. For initial configurations with sufficiently small Rossby numbers the QG modon did not change, while for large Rossby numbers (typically 0.2 ) it evolved towards a cyclone-anticyclone asymmetric ageostrophic coherent structure (let us recall that the QG modon is a perfectly symmetric dipole).

In the present work we report a discovery of an essentially ageostrophic modon $(\mathrm{Ro}=0.4)$ which is bound to a hydraulic jump (shock) situated at its center. This structure is essentially asymmetric, preserves coherence for a long time (compared to the turnover period of each vortex component), and moves along a circular path. Such "shock modon" appears as a result of relaxation of the QG modon with large Ro. As in our previous papers on ageostrophic modons [8], [9] we work with a newgeneration finite-volume code for RSW equations, which is well balanced (i.e., preserves equilibrium solutions) and entropy satisfying (i.e. guarantees the energy decrease across shocks) [10]. The spatial resolution is 0.025 in units of the deformation radius $R_{d}=\sqrt{g H_{0}} / f$ ( $g$ is gravity and $H_{0}$ is unperturbed thickness), which is sufficient to perform a correct investigation of fine-scale structures, like shocks.

It should be kept in mind that RSW equations are equivalent to the equations of dynamics of a barotropic gas in the presence of the Coriolis force in two dimensions with two components of velocity $\mathbf{v}=(u, v)$ and thickness $h$ as dynamical variables. The invariant characteristic of the vortex is potential vorticity (PV) $q=\frac{\hat{\mathbf{z}} \cdot(\nabla \wedge \mathbf{v})+f}{h}$, or its anomaly with respect to the background value $q-f / H_{0}$.
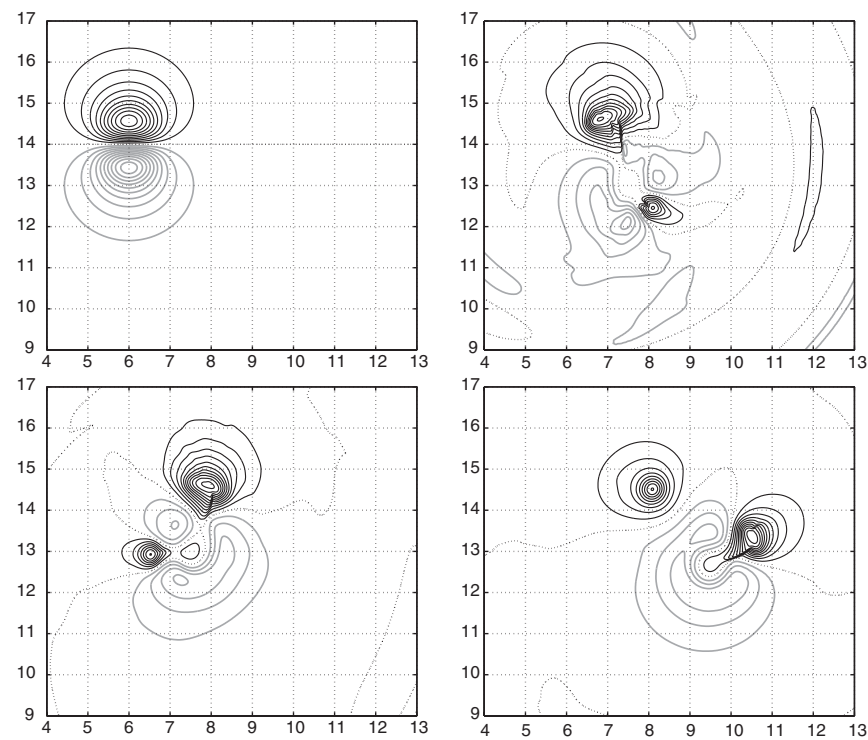

FIG. 1. Evolution of thickness during relaxation of the QG modon with Ro $=0.4$. From left to right and top to bottom: $t=$ $0,6,12$, and 24 (units of $f^{-1}$ ). The thickness anomaly is given at the interval 0.06 in units of $H_{0}$. Black: cyclone (depression), gray: anticyclone. Thin dotted line indicates zero thickness anomaly. 


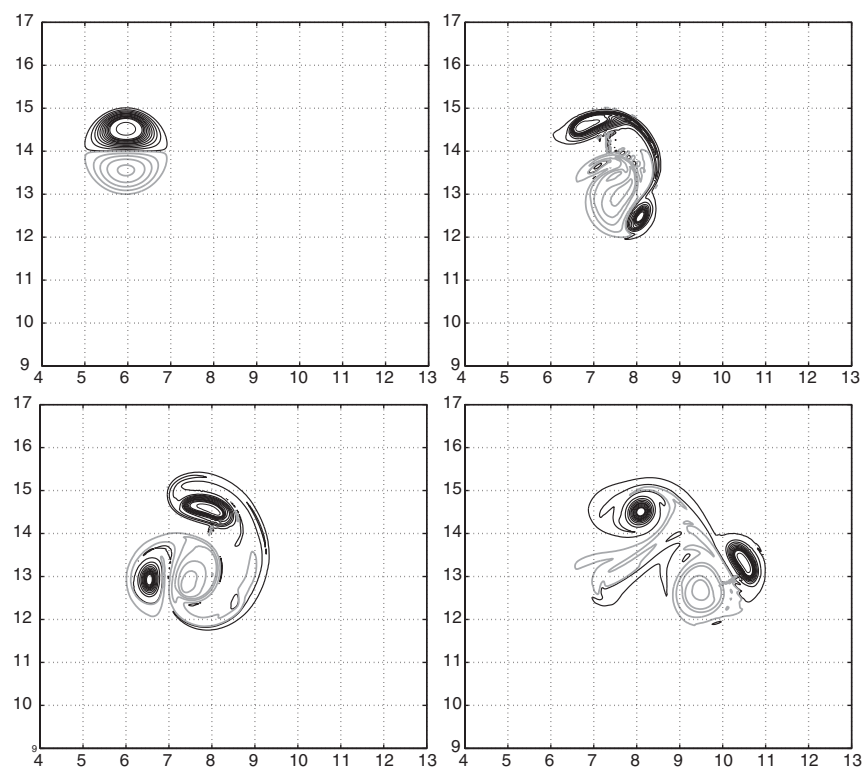

FIG. 2. Evolution of the PV anomaly corresponding to Fig. 1. Values from \pm 0.1 [respectively in black $(+)$ and gray $(-)$ ] at the interval 1 up to 10.1 and then at the interval 5 (suitable for cyclone only), in units of $\frac{f}{H_{0}}$.

The initial stages of the adjustment of a QG modon with large Ro are presented in Fig. 1 (pressure), and Fig. 2 (potential vorticity). Only a part of the computational domain is shown, the full size being $20 \times 20 R_{d}^{2}$, and Neumann (sponge) boundary conditions are used. Unlike more "gentle" relaxation observed for smaller Rossby numbers [8], where the initial dipole was adjusting to the new equilibrium shape by emitting inertia-gravity waves and ejecting weak cyclonic PV filaments, one sees here a massive initial ejection of cyclonic PV advected by the anticyclone (see Fig. 2 at $t=6$ ), and emission of largeamplitude waves (visible in Fig. 1 at $t=6$ ), mostly absorbed by the sponges. This transient process leads to an asymmetric dipole, together with an isolated cyclone, which keep their coherence. At $t=40$, the monopolar cyclone is removed from the computational domain and boundary conditions are replaced with energetically neutral periodic ones, in order to follow the proper evolution of the dipole.

The distributions of thickness/velocity, PV, and divergence for the resulting dipolar vortex at $t=46$ are given in Fig. 3. One clearly sees the hydraulic jump both in the thickness distribution (left panel), and in the divergence field (right panel). This is confirmed by Fig. 4, where we plot the profiles of thickness, divergence, and modulus of velocity along the dipole's axis. A typical hydraulic jump, with velocity decreasing and thickness increasing across the jump, is recognizable in the figure (the mean flow is from right to left). We recall that inertia-gravity waves are equivalent to sound waves, following the above-mentioned gas dynamics analogy. Their typical velocity is of order $\sqrt{g H_{0}}$, so that the hydraulic jump corresponds to the transition from transcritical (maximal Froude number 1.1-1.2) to subcritical flow.

Evolution of the energy of the system is presented in Fig. 5 together with the evolution of the energy dissipation, both in nondimensional units. We recall that the numerical scheme integrates the RSW equations in the conservative form, cf. [10], and has no explicit dissipation. The numerical dissipation is calculated as the departure of the discrete energy balance in each grid cell summed over all cells. The built-in property of the code is that dissipation is positive cellwise and that energy decreases across the shocks.

The shock vanishes after $\approx 70$ inertial periods, which is correlated with the stabilization of the dissipation rate (see Fig. 5, lower panel). The well-formed modon persists for a long time afterwards (not less than 460 inertial periods), slowly losing intensity and moving along an almost perfect circular path (cf. Figure 6). The characteristics of this modon are similar to those observed for the modons at smaller Rossby number in [8]. During this time, and despite the absence of hydraulic jump, the maximal Froude number remains very close to 1 . It decreases from 1.2 at $t=40$ to 0.95 at $t=460$. The slow energy decrease
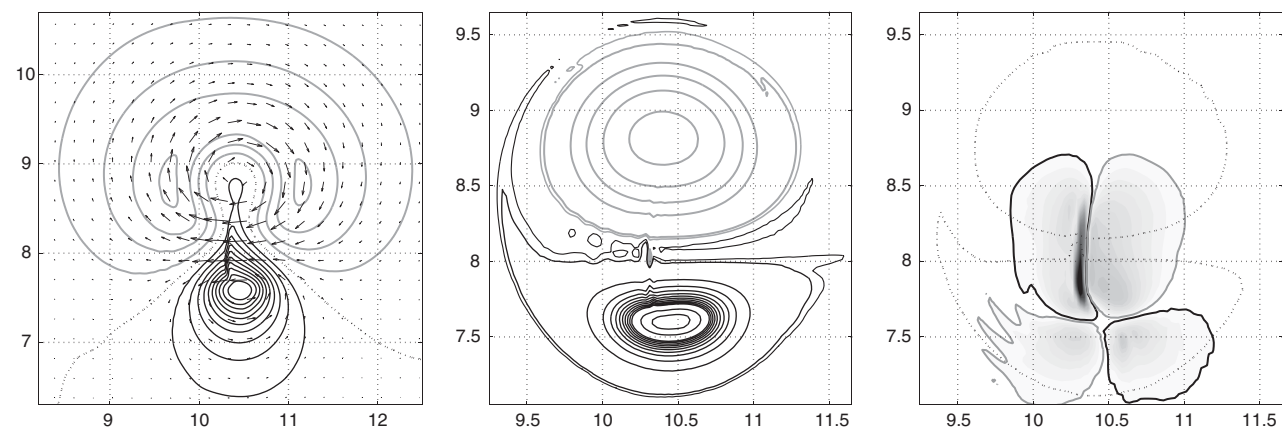

FIG. 3. Isobars with superimposed velocity field (left), PV anomaly (middle), and divergence (right) of the modon at $t=46$. Same legend as in Figs. 1 and 2 for the left and middle panels. Thin lines are added at values \pm 0.5 for the PV anomaly. Thin black dotted lines in the right panel indicate PV anomaly $q_{A}= \pm 0.1$ contours roughly corresponding to the dipole's edge. Absolute value of divergence in units of $f$ : levels of gray from 0.1 (white) to 4 (black); Limits of convergence (divergence) zones: black (gray) contours. 


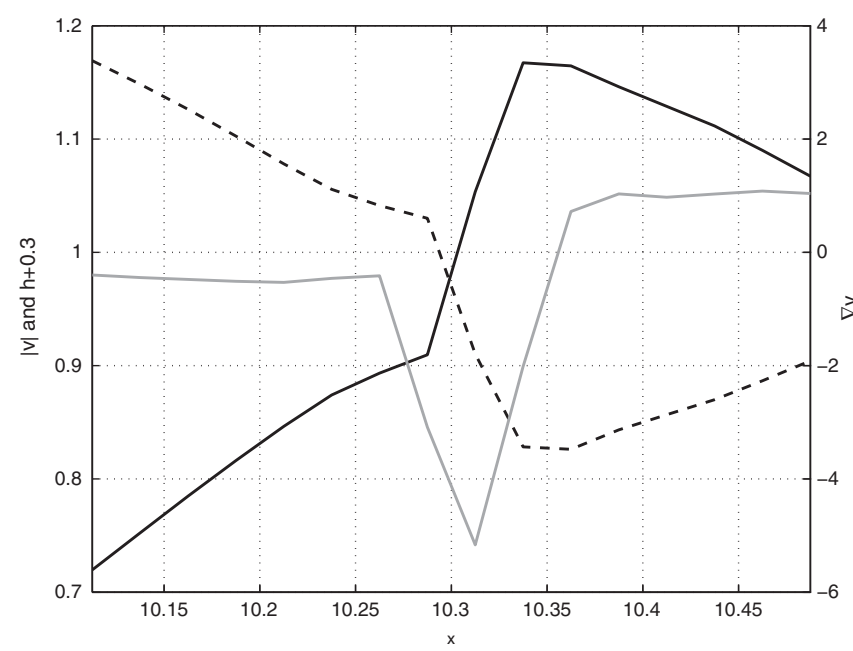

FIG. 4. $|\mathbf{v}| / \sqrt{g H_{0}}$ (Froude number, black line), thickness $h+0.3$ (dashed line), and divergence $\nabla \cdot \mathbf{v}$ (gray line) profiles along the modon's axis at $t=46$.

(cf. Figure 5) is due to numerical dissipation. No wave radiation was observed at this stage.

Let us recall for steady-moving structures in RSW, the Bernoulli function: $\quad B=\frac{1}{2}(u-\bar{U})^{2}+\frac{1}{2}(v-\bar{V})^{2}+g h+$ $\bar{U} f y-\bar{V} f x$, where $(\bar{U}, \bar{V})$ is the velocity of the structure, and PV are functionally dependent [11], which should be reflected in the corresponding scatter plot. Such plot for the shock modon is presented in Fig. 7.

As compared to the scatter plots for ageostrophic modons at smaller Rossby numbers [8], this one is less sharp, especially in the cyclonic (positive PV) part. This is explained by the presence of the hydraulic jump which is situated mostly in the cyclonic part of the dipole, cf. Fig. 3, left panel. $B$ inherits the jump in $h$, while the jump in PV is possible only if $B$ varies along the shock (e.g., if the latter
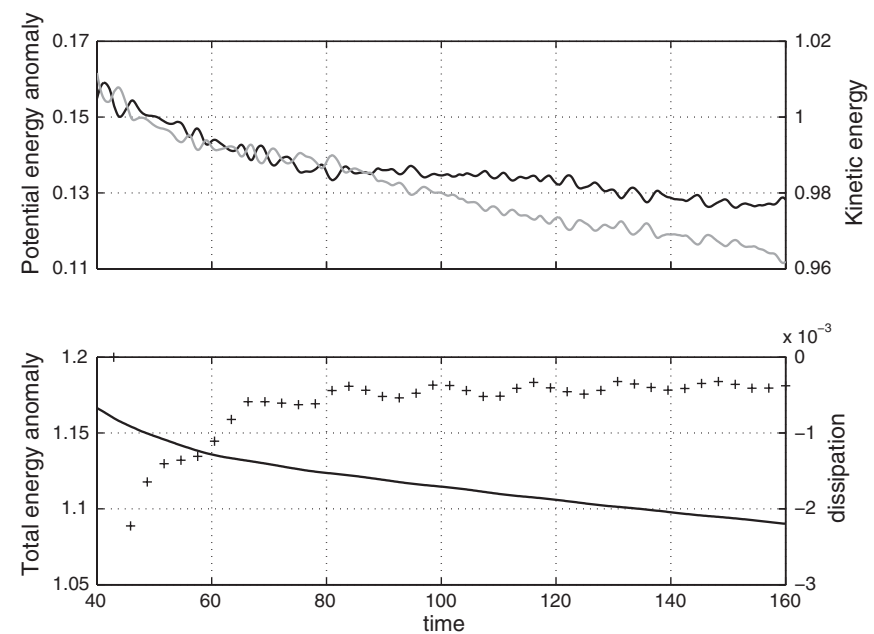

FIG. 5. Upper panel: kinetic (gray) and potential (black) energy during the evolution of a shock modon. Lower panel: total energy (continuous line) and energy dissipation (crosses).

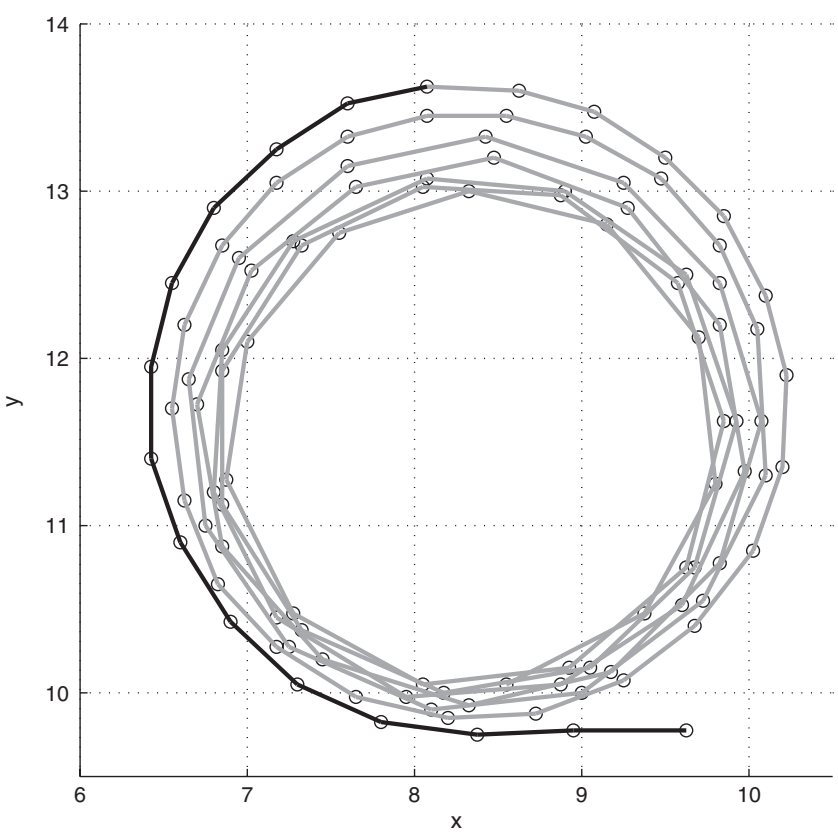

FIG. 6. Modon trajectory from $t=40$ to $t=460$. Color switches from black to gray when the shock vanishes. Time interval: 5 .

is curved [12]). The signature of the shock in PV is negligible, and for each value of PV a range of values of $B$ within the jump appears. Another source of the spread is uncertainties in the computed Bernouilli function, especially when the trajectory is curved, as above. Nevertheless, the scatter plot is sharp enough to assert, together with the long lifetime, the coherence of the shock modon.

To test the robustness of the shock modons, we performed a number of simulations of their collisions, similar to our previous study [9]. They show that shock modons

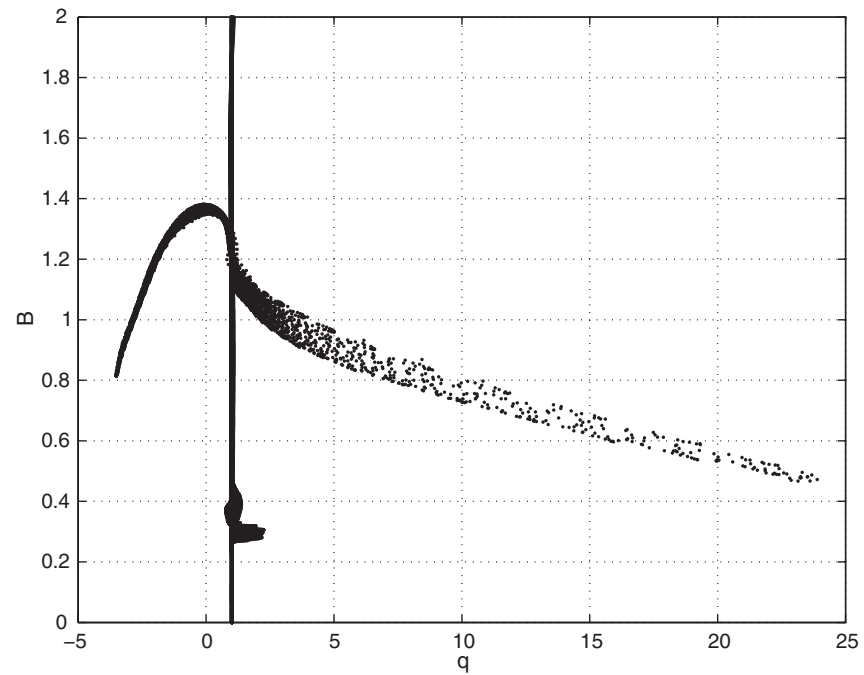

FIG. 7. Bernoulli function vs PV for the shock modon at $t=46$. 

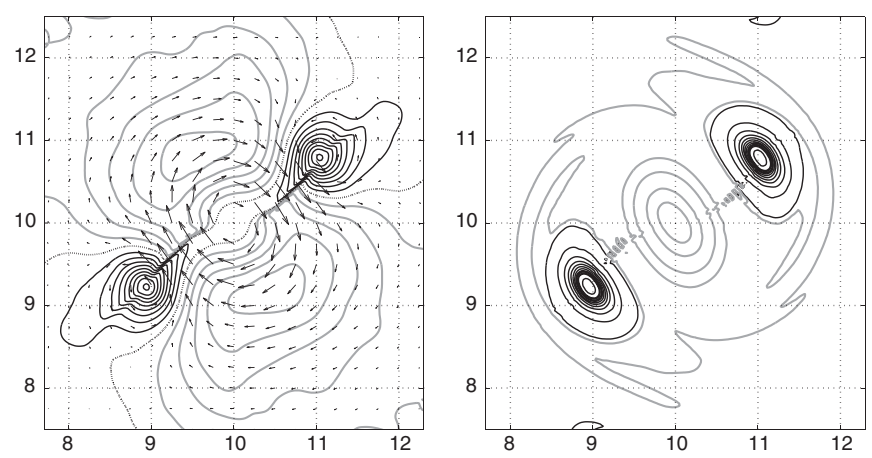

FIG. 8. Isobars with superimposed velocity field (left) and potential vorticity anomaly (right) of the "shock tripole" formed after the collision of two shock modons. Same legend as in Figs. 1 and 2.

undergo frontal collisions forming either a new pair of shock modons after partner exchange, or a "shock tripole" with a fusion of anticylonic vortices. The latter contains two hydraulic jumps situated between the anticyclonic core and cyclonic satellites (cf. Figure 8).

Thus, geostrophic modons with a large Rossby number in rotating shallow water evolve towards a new quasisteady coherent structure, shock modon which is a combination of an asymmetric vortex dipole and a hydraulic jump. The structure is dissipative, like the hydraulic jump (shock) itself, yet long living and robust with respect to the interactions with other vortices, as follows from the preliminary study of their collisions. Collisions of shock modons can also produce other coherent shock vortices, like a tripole, indicating that shock-vortex structures are universal at large Rossby numbers in rotating shallow water. We expect that these kinds of solutions will be relevant for astrophysical generalizations of the model mentioned above.

This work was supported by the French ANR grant SVEMO.

*zeitlin@1md.ens.fr

[1] J. Pedlosky, Geophysical Fluid Dynamics (Springer, New York, 1987).

[2] P. Gilman, Astrophys. J. Lett. 544, L79 (2000).

[3] O. Umurhan, Astron. Astrophys. Rev. 489, 953 (2008).

[4] K. Mima and A. Hasegawa, Phys. Fluids 21, 81 (1978).

[5] V. Larichev and G. M. Reznik, Dokl. Akad. Nauk SSSR 231, 1077 (1976).

[6] V. M. Kamenkovich, G. M. Reznik, in Fizika okeana. V.2. Gidrodinamika okeana (in Russian) (Nauka, Moscow, 1978).

[7] Z. Kizner, G. M. Reznik, B. Fridman, R. Khvoles, and J. McWilliams, J. Fluid Mech. 603, 305 (2008).

[8] B. Ribstein, J. Gula, and V. Zeitlin, Phys. Fluids 22, 116603 (2010).

[9] N. Lahaye and V. Zeitlin, Phys. Fluids 23, 061703 (2011).

[10] F. Bouchut, in Nonlinear Dynamics of Rotating Shallow Water: Methods and Advances, edited by V. Zeitlin (Elsevier, Amsterdam, 2007).

[11] P. Malanotte-Rizzoli, Adv. Geophys. 24, 147 (1982).

[12] J.M. Lighthill, Waves in Fluids (Cambridge University Press, Cambridge, England, 1978). 